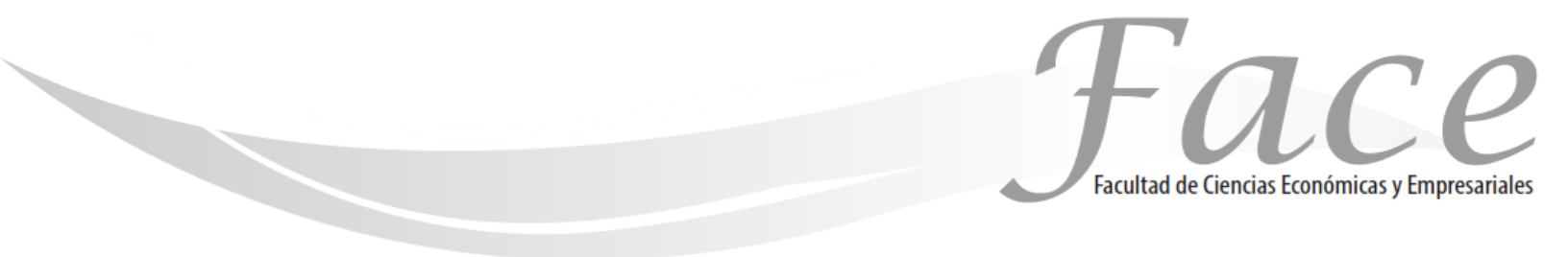

ISSN Impreso: 1794-9920 ISSN Electrónico: 2500-9338

Volumen $16-\mathrm{N}^{\circ} 2$

Año 2016

Págs. 54 - 65

\title{
EL MÉTODO DE COSTOS ESTÁNDAR ALINEADO A PEQUEÑAS INDUSTRIAS MANUFACTURERAS DEL ÁREA METROPOLITANA DEL VALLE DE ABURRÁ
}

\author{
José Luis Cardona Olaya* \\ Enlace ORCID: http://orcid.org/0000-0002-0325-0967 \\ Cristian Pabón Salazar** \\ Enlace ORCID: https://orcid.org/0000-0003-0059-493X \\ Sandra Milena Velásquez Restrepo*** \\ Enlace ORCID: http://orcid.org/0000-0002-6697-2801 \\ Carolina Abril Carrascal ${ }^{* * * *}$ \\ Enlace ORCID: http://orcid.org/0000-0002-7183-1915 \\ Lesly Lisbeth Gómez Echeverry \\ Enlace ORCID: http:// orcid.org/0000-0003-3344-0726
}

Fecha de Recepción: 7 de Octubre 2016

Fecha de Aprobación: 20 de Diciembre 2016

\section{Resumen:}

Las empresas manufactureras demandan en sus procesos los costos de fabricación de sus productos, los cuales podrían obtenerse a partir del método de costos estándar; sin embargo, no lo utilizan debido a su desconocimiento. Con el propósito de ilustrar al sector manufacturero de calzado y marroquinería sobre su utilización, se elaboró un modelo para el cálculo y el tratamiento de los costos estándar, partiendo de las normas internacionales asociadas a él. Se concluyó que este método es una herramienta útil, que permite construir eficientemente los estados financieros de una empresa y contribuye a tomar decisiones estratégicas.

Palabras Claves: Método de Costos estándar, hoja de cálculo, pequeñas empresas, manufactura

\footnotetext{
* Contador Público, Magíster en Gestión Contable y Financiera; Contacto: abanicoempresarial@gmail.com

* * Aprendiz en Tecnología de Contabilidad y Finanzas; Contacto: crixtianpx97@hotmail.com

* * * Bioingeniera, Magíster en Ingeniería, MBA, Especialista en Gerencia; Contacto: smvelasquez@sena.edu.co

* * * * Ingeniera de Materiales, Magíster en Gestión de Ciencia, Tecnología e Innovación; Contacto: cabril64@misena.edu.co

* * * * Bioingeniera; Contacto: Igomeze@sena.edu.co
} 


\title{
THE STANDARD COST METHOD FOR SMALL MANUFACTURING INDUSTRIES OF THE METROPOLITAN AREA OF THE ABURRÁ VALLEY
}

\begin{abstract}
:
Manufacturing companies require a clear cost manufacturing process, which could be obtained from the standard cost method; however, it is not used due to the companies' lack of knowledge. In order to illustrate the use of this method in the footwear and leather manufacturing industry, a model was developed for the calculation and treatment of standard costs based on international standards. It was therefore concluded that the above-mentioned method is a useful tool, which allows the companies to efficiently prepare their financial statements and help them make strategic decisions.
\end{abstract}

Keywords: Standard cost method, Spreadsheet, Small companies, Manufacturing.

\section{O MÉTODO DE CUSTOS PADRÃO PARA PEQUENAS INDÚSTRIAS MANUFATUREIRAS DA ÁREA METROPOLITANA DO VALE DO ABURRÁ}

\begin{abstract}
Resumo:
As empresas manufatureiras requerem em seus processos os custos de fabricação de seus produtos, os quais se podem obter a partir do método de custo padrão; no entanto, não o utilizam devido a seu desconhecimento. Com o propósito de ilustrar ao sector manufatureiro de calçado e couro sobre sua utilização, elaborou-se um modelo para o cálculo e o tratamento dos custos padrão partindo das normas internacionais associadas a ele. Concluiu-se que este método é uma ferramenta útil, que permite elaborar eficientemente os estados financeiros de uma empresa e contribui para um processo de tomada de decisões estratégicas.
\end{abstract}

Palavras-Chave: Método de custo padrão, folha de calculo, pequenas empresas, manufatura. 


\section{INTRODUCCIÓN:}

La contabilidad de costos es una rama de la contabilidad que tiene como objetivo establecer el costo asociado a la realización de un producto, controlando cada una de las fases del proceso productivo; sin embargo, en las empresas manufactureras se deben considerar una gran cantidad de variables que dificultan la identificación del costo real del producto. Dentro de la contabilidad de costos se encuentra el sistema de costeo estándar, que facilita la manipulación de estas variables y calcula el costo de cada producto, sin dejar de lado la optimización de tiempos y materias primas.

El método de costos estándar es ampliamente utilizado por las empresas manufactureras de diferentes países (Horngren, Foster \& Datar, 2007)(ver Tabla 1).

Tabla 1.

Porcentaje de uso del método de costos estándar en diferentes países.

\begin{tabular}{cc}
\hline PAís & PORCENTAJE \\
\hline Australia & $92 \%$ \\
\hline Japón & $90 \%$ \\
\hline Irlanda & $87 \%$ \\
\hline China & $87 \%$ \\
\hline Estados Unidos & $76 \%$ \\
\hline Nueva Zelanda & $73 \%$ \\
\hline India & $68 \%$ \\
\hline Singapur & $56 \%$ \\
\hline Reino Unido & $90 \%$ \\
\hline
\end{tabular}

Fuente: adaptación de Horngren, Foster \& Datar, 2007

Para determinar el grado de uso de los costos estándar en las empresas manufactureras colombianas, un estudio indagó a 35 empresas que representan el $40 \%$ de los ingresos totales de Antioquia, y el 8,6\% de Colombia, pertenecientes a varios sectores (Duque, Osorio, \& Agudelo, 2012). El resultado reflejó un desconocimiento generalizado del método de costos estándar y que sólo el $14 \%$ de las empresas lo utiliza en su sistema contable.

Al estudiar el sector del cuero, calzado y su manufactura, se encontró la insuficiencia de información proyectada, ajustada al tiempo real mediante el uso de los costos estándar (Madegowda, 2007) y de los presupuestos flexibles. Es por esto que fue necesario crear un modelo en el cual se presenta una hoja de cálculo por producto, con las respectivas indicaciones para su uso (Checo Paulino, 2000). Se espera que las empresas que implementen este modelo evidencien su evolución y cuenten con la información necesaria que permita tomar decisiones estratégicas de manera certera, para contribuir a su sostenibilidad en el mercado y a superar los desafíos que este les impone.

En este artículo se encontrará un breve marco teórico referente a la contabilidad y al método de costos estándar; por otra parte, se describirá la metodología utilizada, que consta de una revisión bibliográfica seguida de un sondeo a profesionales contadores, la información adquirida sirvió de insumo para el diseño de una plantilla de cálculo de los costos estándar y luego se efectuó una encuesta para identificar el conocimiento y apropiación del método a la industria manufacturera del cuero, calzado y marroquinería; además se encontrará la percepción acerca de la implementación del modelo en una de las empresas encuestadas. Para finalizar, se apreciarán algunas conclusiones relacionadas al método descrito.

\section{MARCO TEÓRICO:}

Los costos se definen como "las erogaciones y cargos realizados para producir los bienes o prestar los servicios de los cuales un ente económico ha derivado sus ingresos" (Artículo 39, 1993); es decir, es lo necesario para obtener un bien, sea producto o servicio y disponerlo a la venta. La contabilidad de costos clasifica, acumula, controla y asigna los costos. La información de los costos permite establecer el precio de los productos, controlar las operaciones y desarrollar estados financieros. Los costos se determinan considerando las materias primas (cantidad y precio), la mano de obra (cantidad de horas y estándar salarial por hora) y los costos indirectos de fabricación (fijos y variables) (Salguero, 2011).

Existen diferentes métodos que llevan a calcular los costos, entre ellos se encuentra el sistema de costos estándar, que sirve para medir una actuación real (Duque, Osorio\& 


\section{EL MÉTODO DE COSTOS ESTÁNDAR ALINEADO A PEQUEÑAS INDUSTRIAS MANUFACTURERAS DEL ÁREA METROPOLITANA DEL VALLE DE ABURRÁ}

José Luis Cardona Olaya - Cristian Pabón Salazar - Sandra Milena Velásquez Restrepo - Carolina Abril Carrascal - Lesly Lisbeth Gómez Echeverry

Agudelo, 2012). Se debe revisar la normatividad aplicable a la producción de bienes y servicios, para obtener una evaluación apropiada y realista del costo. Para cada producto fabricado se prepara una ficha de costo estándar, que muestra tanto el costo de cada proceso como el costo acumulativo (Salguero, 2011).

La palabra gasto representa el flujo de salida de los recursos, en forma de disminuciones del activo 0 incrementos del pasivo o una combinación de ambos (Artículo 40, 1993). En Colombia se ha hecho una fiel separación de los costos y gastos, haciendo referencia a costo en solo lo relacionado a la fabricación, o prestación del servicio y gasto a las ayudas económicas necesarias para terminar el proceso de enajenación. En esta definición se incluyen los costos en manufactura, mercadeo, distribución y ventas (Gómez, 2005). Sin embargo, en las culturas inglesa y española por ejemplo, las palabras costos y gastos hacen referencia al mismo concepto, dónde se incluye en los costes del periodo los gastos de personal (García, 2015). Por otra parte, en países como India se opta por incluir los costos de investigación y desarrollo I+D en los costos asociados a la fabricación de productos; algo que en Colombia todavía no se contempla (Strengthening cost competitiveness, 2012).

Este método de costos estándar es uno de los más utilizados por las empresas manufactureras en el mundo (Rao, Kashanni\& Marie, 2010) debido a que presenta una enorme eficiencia a costos mínimos. Estudios realizados revelan que de 231 empresas encuestadas en Reino Unido, más del $90 \%$ de estas aplican el costeo estándar para el control de sus costos (Horngren, Foster \& Datar, 2007). En Colombia, se desarrolló un proyecto de investigación que tenía como propósito diseñar e implementar un sistema de costos estándar a una empresa manufacturera del sector textil y confecciones. Luego de ejecutado el proyecto se encontró que este sistema brinda a la empresa la posibilidad de generar productos con mayor calidad y economía en la producción, mejorando su competitividad; además, se identificó la necesidad de crear un departamento de costos que suministre la información de forma oportuna y real sobre los costos de todas las áreas de la empresa (Salguero, 2011).

\section{METODOLOGÍA:}

Para abordar correctamente el método de costos estándar, se realizó una revisión bibliográfica del tema, lo que permitió extraer los elementos teóricos necesarios para plantear su adaptación a los requerimientos de las empresas colombianas. Paralelamente, se efectuó un sondeo a profesionales contadores, quienes aportaron sus apreciaciones y experiencia respecto al uso de este método. Como principal insumo de esta revisión, se identificaron los parámetros más relevantes de la norma internacional de contabilidad, ajustándolos luego a la realidad colombiana ("Norma Internacional de Contabilidad 2. NIC 2," s.f.); luego se diseñó el formato de costos estándar, incluyendo las ecuaciones pertinentes para lograr su cálculo. Con el propósito de verificar el nivel de conocimiento e implementación del método de costos estándar en las empresas, se enfocó el estudio al sector manufacturero de calzado y marroquinería del valle de Aburrá. Se seleccionó un grupo de empresas teniendo en cuenta la base de datos de la Asociación Colombiana de Industriales de Calzado, Cuero y su Manufactura ACICAM.

El tamaño de la muestra se determinó mediante el teorema del límite central (ver Ecuación 1), el cual especifica que, cuando las condiciones son muy generales, la suma de muchas variables aleatorias independientes se aproximan a una distribución normal, también llamada campana de Gauss (Ochoa, 2013):

\section{Ecuación $n^{\circ} 1$.}

Teorema del límite central.

$$
\mathrm{n}=\frac{N Z^{2} \mathrm{p}(1-\mathrm{p})}{(\mathrm{N}-1) \mathrm{e}^{2}+\mathrm{Z}^{2} \mathrm{p}(1-\mathrm{p})}
$$

Dónde: $\mathrm{n}=$ tamaño de la muestra; $\mathrm{N}=$ tamaño del universo; $Z=$ desviación del valor medio, tomando la distribución de Gauss, los valores más frecuentes para un nivel de confianza del $95 \%, Z=1.96$; e = margen de error máximo admisible; $p=$ proporción (como no se tiene información se elige el menos favorable de los casos, donde $p=50 \%$ ). Se aplica el teorema de límite central para determinar el tamaño de la muestra a partir de la base de datos proporcionada por ACICAM, la cual registra 60 empresas en el valle de Aburrá; entonces se tiene: $N=60 ; Z=1.96$; e $=5 \%$ y $p=50 \%$

$$
\begin{gathered}
n=\frac{60 * 1.96^{2} * 0.5(1-0.5)}{(60-1) 0.05^{2}+1.96^{2} 0.5(1-0.5)} \\
=52,012 \approx 52
\end{gathered}
$$

Por lo tanto, para un nivel de confianza del $95 \%$ y un error máximo del $5 \%$, la encuesta se realizó a 52 empresas.

Una vez establecido el tamaño de la muestra, se diseñó y elaboró una encuesta para determinar el nivel de conocimiento de las empresas respecto a este tema e identificar su grado de interés y disposición para implementar este método. Las encuestas se efectuaron personalmente, por vía telefónica y correo electrónico.

Posteriormente se eligió una empresa del grupo encuestado en la que se llevó a cabo la prueba piloto, 
aplicando el método de costos estándar. Se desarrollaron 3 reuniones para establecer los parámetros a medir, se implementó el método y para finalizar se estableció un comparativo del resultado obtenido con el método de la empresa y la hoja de cálculo de costos estándar desarrollada en este proyecto.

Finalmente, para determinar la percepción obtenida en la ejecución de este proyecto, se llevó a cabo una encuesta de satisfacción a la empresa donde se desarrolló la prueba piloto.

\section{RESULTADOS:}

\subsection{Nivel de conocimiento sobre el Método de Costos Estándar}

Inicialmente, se ratifica que todas las empresas seleccionadas coinciden en la importancia de conocer el costo real del producto que fabrican (ver Figura 1); sin embargo, un poco menos de la mitad de estas (ver Figura 2) cuentan con un área de costos para poderlo determinar.

\section{Figura 1.}

Importancia sobre conocimiento el costo del producto.

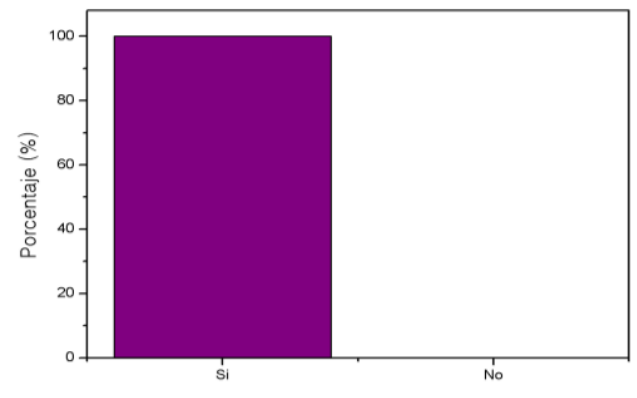

Fuente: Elaboración propia.

Figura 2.

Existencia del área de costos en la empresa. Fuente: Elaboración propia.

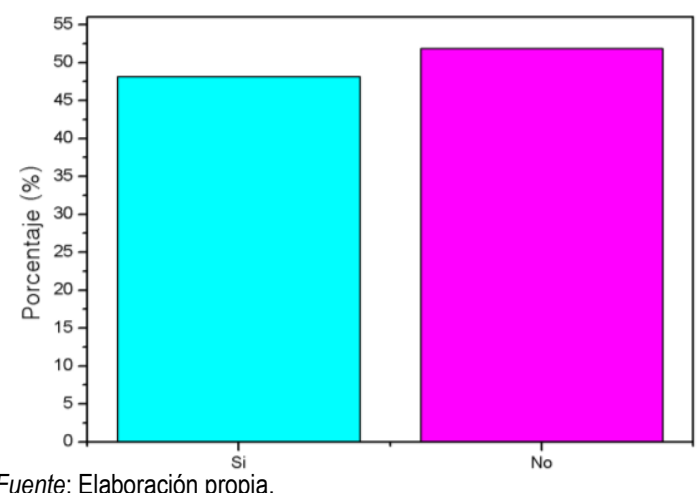

Fuente: Elaboración propia.
En la figura 3, se encuentran los diferentes métodos bajo los cuales las empresas manufactureras encuestadas calculan el costo real de sus productos.

\section{Figura 3.}

Manejo de los costos por parte de la empresa.

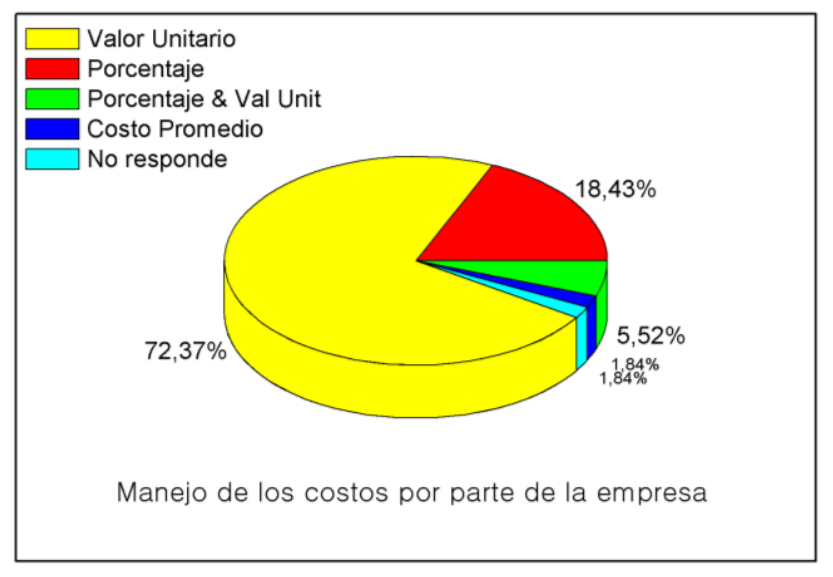

Fuente: Elaboración propia.

Como se evidenció en la figura anterior, no fue incluido el método de costos estándar; esto puede deberse al poco conocimiento de este método en el sector del Calzado, Cuero y su Manufactura (ver Figura 4). Este resultado refuerza la hipótesis planteada inicialmente, donde se expone que este tipo de empresas no aplican el método por desconocimiento.

\section{Figura 4.}

Conocimiento del método de costos estándar en la empresa.

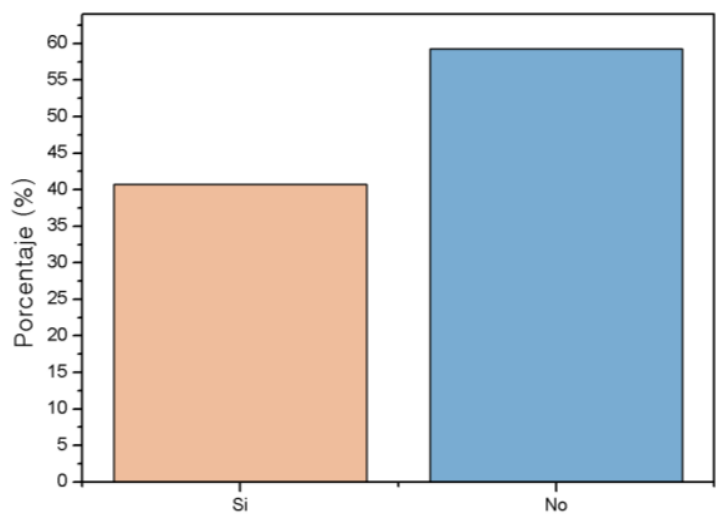

Fuente: Elaboración propia. 


\section{EL MÉTODO DE COSTOS ESTÁNDAR ALINEADO A PEQUEÑAS INDUSTRIAS MANUFACTURERAS DEL ÁREA METROPOLITANA DEL VALLE DE ABURRÁ}

José Luis Cardona Olaya - Cristian Pabón Salazar - Sandra Milena Velásquez Restrepo - Carolina Abril Carrascal - Lesly Lisbeth Gómez Echeverry

Sin embargo, la mayoría de las empresas están dispuestas a implementar esté método con ayuda del Servicio Nacional de Aprendizaje, como se aprecia en la figura 5.

\section{Figura 5.}

Disposición para la implementación del método de costos estándar en conjunto con el SENA.

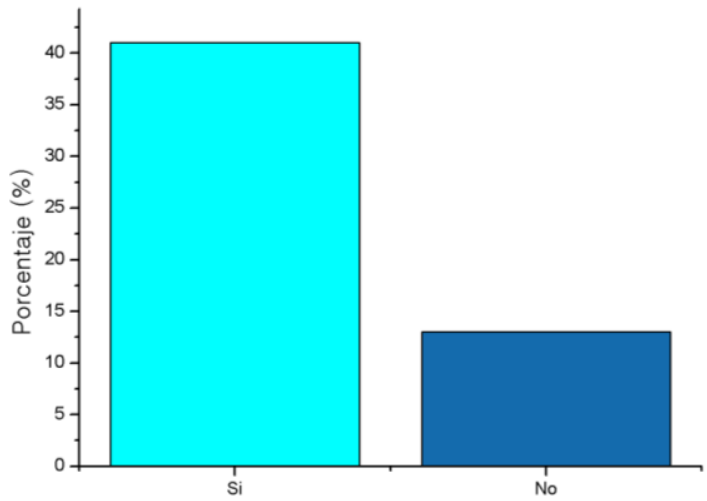

Fuente: Elaboración propia.

\subsection{Diseño de la hoja de cálculo para el Método de Costos Estándar}

A continuación se presenta la tabla 2, donde se condensa la información necesaria para calcular los costos estándar al interior de una empresa manufacturera:

Tabla 2.

Elaboración de los Costes estándar.

\begin{tabular}{|c|c|c|c|}
\hline ELEMENTO & QUE & PUE / SUE & CUE \\
\hline MP & $\begin{array}{l}Q U E_{m p} \\
=\frac{\text { Elemento }}{\text { Unidad }}\end{array}$ & $\begin{array}{l}\text { PUE } \\
=\frac{\text { Pesos }}{\text { Elemento }}\end{array}$ & $\frac{\text { Pesos }}{\text { Unidad }}$ \\
\hline MOD & $\begin{array}{l}Q U E_{\text {mod }} \\
=\frac{\text { tiempo }}{\text { Unidad }}\end{array}$ & $S U E=\frac{\text { Pesos }}{\text { tiempo }}$ & $\frac{\text { Pesos }}{\text { Unidad }}$ \\
\hline \multirow[t]{2}{*}{ CIF } & $\begin{array}{l}Q U E_{\text {cif }} \\
=\frac{\text { Tiempo }}{\text { Unidad }}\end{array}$ & $\begin{array}{l}\text { TPCif } \\
=\frac{\text { Pesos }}{\text { Tiempo }}\end{array}$ & $\frac{\text { Pesos }}{\text { Unidad }}$ \\
\hline & & TOTAL & $\sum$ CUE \\
\hline
\end{tabular}

Frente a los materiales necesarios, es decir la cantidad unitaria estándar (QUE) de materiales, se debe hacer un estimado de las cantidades necesarias de acuerdo a la demanda estándar por unidad de fabricación. Al respecto hay dos formas de hacer el cálculo, uno basado en la experiencia y otro hecho matemáticamente.

De acuerdo a la experiencia del fabricante, se podría suponer un elemento $X$ que se vende por libras, del cual se obtienen $Y$ unidades de producto. En este caso, el cálculo se deberá realizar de la siguiente manera:

\section{Ecuación 2.}

Cantidad Unitaria Estándar.

$$
\mathrm{QUE}_{\mathrm{MP}}=\frac{\mathrm{X}_{\text {libras }}}{\mathrm{Y}_{\text {unidades }}}=\frac{\text { libra }}{\text { unidad }}
$$

Sin embargo, al momento de aplicarlo a grandes cantidades, es conveniente identificar las capacidades requeridas por la empresa para fabricar una unidad. Otra forma de hacer el cálculo, es cuantificando cada uno de los elementos necesarios para elaborar el producto, en este caso se requiere de técnicas específicas enfocadas al producto.

Respecto a la mano de obra se debe cuantificar cada uno de los procesos, los métodos y tiempos de fabricación o de traslación; lo cual requiere de un análisis claro y profundo. En caso de no tener acceso a dicha información, se podría recurrir a la Ecuación 3, utilizando el tiempo total transcurrido y el número de unidades fabricadas en dicho periodo de tiempo.

\section{Ecuación 3.}

Análisis de métodos y tiempos.

$$
\frac{\text { Zhoras }}{\text { Yunidades }}=\frac{\text { Hora }}{\text { Unidad }}
$$

Para obtener el costo de la unidad, se deben dividir las $Z$ horas, que corresponden al tiempo empleado por la persona sobre el número promedio $\mathrm{Y}$ de unidades que se logran fabricar. El tiempo será expresado en horas y este puede variar dependiendo del método utilizado. En esta investigación no se tomó en cuenta debido a que no es objeto de estudio.

Ahora bien, también se deben tener presentes los costos indirectos, los cuales afectan la producción, pero no se asignan directamente al producto. Hay dos tipos de costos indirectos: fijos y variables. Los costos fijos permanecen 
constantes ante cualquier fluctuación del volumen de producción, mientras que los costos variables fluctúan de manera proporcional a estos cambios. La sumatoria de estos dos da como resultado los costos indirectos (Universidad Eafit, s.f.).

La relación de los costos indirectos a la producción se calculará dependiendo del factor dominante, para determinarlo se debe tener en cuenta la base presupuestal. Existen varios métodos de medición: a) basado en las horas de Mano de Obra, MOD, b) basado en las horas máquina y c) basado en las unidades estimadas de producción (Sinisterra, 2006).

Dado esto, se deberá realizar un presupuesto de costos indirectos de fabricación CIF variables y otros fijos y dividirse por el total de horas presupuestadas de MOD, por el total de horas maquina presupuestadas 0 por las unidades presupuestadas.

\section{Ecuación 4.}

Costos indirectos de fabricación.

$$
\frac{\text { Totalhoraspresupuestadas }}{\text { unidadespresupuestadas }}
$$

Es conveniente recordar que el total de horas puede referirse a horas de mano de obra o ejecutadas por una máquina y que las unidades serán expresadas en un porcentaje.

En la columna correspondiente a los PUE/SUES, se encuentra el precio promedio de adquisición de algo durante el año; en caso de ser variable se deberá optar por el mayor valor. Las diferentes circunstancias permiten deducir una variabilidad en todos los casos.

\subsubsection{Hoja de Costos Reales}

La hoja de costos reales se deberá utilizar de acuerdo a la contabilidad real de la empresa, se sugiere que esta se realice por producto y bajo sus estándares. Se podrían utilizar estrategias como los centros de costos para determinar costo del producto 0 al finalizar utilizar el costo por producto prorrateando las unidades utilizadas. No es prudente incluir costos ocultos en este método.

En la tabla 3 se expone la hoja de costos reales:
Tabla 3. Hoja de cálculo de costos reales. Fuente:

\begin{tabular}{|c|c|c|c|}
\hline ELEMENTO & CANTIDAD REAL & PUR / SUR & CUR \\
\hline$M P$ & $Q U R_{m p}=\frac{\text { Q.MPreal }}{\text { Und.real }}$ & $\begin{array}{l}\text { PUR } \\
=\frac{\text { MPconsumida }}{Q . \text { MPreal }}\end{array}$ & $\frac{\text { Pesos }}{\text { Unidad }}$ \\
\hline$M O D$ & $Q U R_{\text {mod }}=\frac{T H R}{\text { Und.real }}$ & $S U R=\frac{\text { MODreal }}{\text { THR }}$ & $\frac{\text { Pesos }}{\text { Unidad }}$ \\
\hline CIF & $Q U R_{\text {cif }}=\frac{T H R}{\text { Und.real }}$ & TRCif $=\frac{\text { Cifreales }}{T H R}$ & $\frac{\text { Pesos }}{\text { Unidad }}$ \\
\hline & & TOTAL & $\sum C U R$ \\
\hline
\end{tabular}
Elaboración propia.

\subsubsection{Contabilización}

Según las normas internacionales de contabilidad NIC 2 , es correcto utilizar el método de costo estándar (ver Ecuación 5) en condiciones de eficiencia y capacidad normal, revisando periódicamente las variaciones (ver Tabla 4). No obstante, las pequeñas empresas no cuentan con el recurso humano suficiente que se responsabilice de estos cálculos para hacer el cierre del periodo ("Norma Internacional de Contabilidad 2. (NIC 2)," s.f.).

Ecuación 5.

Costo Estándar

Costoestandar $=$ CUExUndFabricadas

\begin{tabular}{cccc}
\hline CODIGO & CUENTA & DEBITO & CREDITO \\
\hline $1430 \times x$ & INV.PT & $\sum M P(A)+M O D(A)+\operatorname{CIFV}(A)+\operatorname{CIFF}(A)$ & \\
\hline $71 \times x \times 1$ & MP (A) & $C U E_{m p} x$ Und.reales \\
\hline $72 \times x \times 1$ & $\operatorname{MOD}(\mathrm{A})$ & $C U E_{\text {mod }} \times$ Und.reales \\
\hline $73 \times x \times 2$ & $\operatorname{CIFV~(A)~}$ & $C U E_{\text {cifv }} \times$ Und.reales \\
\hline $73 \times x \times 2$ & $\operatorname{CIFF}(\mathrm{A})$ & $C U E_{\text {CifF }} \times$ Und.reales \\
\hline
\end{tabular}

Fuente: Elaboración propia. 


\section{EL MÉTODO DE COSTOS ESTÁNDAR ALINEADO A PEQUEÑAS INDUSTRIAS MANUFACTURERAS DEL ÁREA METROPOLITANA DEL VALLE DE ABURRÁ}

José Luis Cardona Olaya - Cristian Pabón Salazar - Sandra Milena Velásquez Restrepo - Carolina Abril Carrascal - Lesly Lisbeth Gómez Echeverry

Luego de computar el inventario de productos terminados cada uno ellos saldrá con ese registro. El decreto 2649 de 1993 en Colombia, expone un tratamiento básico a través de las cuentas 71,72 y 73 , donde se contabilizan los costos reales y el estándar. Por su parte la norma internacional no determina el tratamiento del plan de cuentas para cada nación, dejándolo a su consideración (Colombia, 1993).

En primer lugar la contabilización del costo de materia prima MP en unidades de producción tiene que ser acorde a la necesidad y al manejo interno, manteniendo el mismo lenguaje; es decir, solicita que antes de hacer alguna solicitud de materiales, el producto tenga la misma unidad de medida que es solicitada (Gómez, 2005), controlado por un KARDEX de MP y valorada por alguno de los dos métodos permitidos (PEPS y PROMEDIO), por lo que la cuenta apropiada para la compra sería de la forma expresada en la tabla 5 :

\section{Tabla 5.}

Cuenta según el tipo de compra.

\begin{tabular}{cccc}
\hline CÓDIGO & CUENTA & DEBITO & CRÉDITO \\
& & & \\
\hline $1405 X X$ & Inventario MP & XXX & \\
& & & \\
\hline $2205 X X$ & Proveedores & & $X X X$
\end{tabular}

Fuente: Elaboración propia.

Con la respectiva entrada al inventario se realizarán las requisiciones manteniendo la nomenclatura, para no perjudicar los métodos de cálculo. De esta manera la contabilización de la requisición estaría dada por (ver tabla $6)$ :

Tabla 6.

Cuentas para la contabilización de la requisición

\begin{tabular}{llll}
\hline CÓDIGO & CUENTA & DEBITO & CRÉDITO \\
\hline $7105 X X$ & MP consumida & XXX & \\
& & & \\
\hline $1405 X X$ & Inventario MP & & XXX \\
& & & \\
\hline Fuente: Elaboración propia. &
\end{tabular}

En segundo lugar, se deben traer a este punto los importes correspondientes a la mano de obra contabilizada habitualmente de acuerdo a la periodicidad de los contratos, y a la capacidad de liquidez para cubrir estos costos; una forma de conocer la periodicidad es saber los cargos de MOD sobre los pasivos en días (ver Ecuación 6).

\section{Ecuación 6.}

Rotación de salarios.

RotaciondeSalarios $=\frac{\text { Obligacionesaempleadosporpagarx } 360}{\text { Gastosdemod }}$

La norma internacional NIC, junto con la Norma Internacional de Información Financiera para pymes NIIF, insisten en el uso de la capacidad normal y el uso del desperdicio de tiempo, que a pesar de ser costoso, no se debe capitalizar dentro del producto (ver Figura 5), tal como lo afirma el Epígrafe 13.13 modulo Inventarios NIIF para pymes: "son ejemplos de costos excluidos de costos de inventarios y reconocidos como gastos en el periodo en el que se incurre los siguientes: a) Importes anormales de desperdicios, MOD y otros costos de producción".

\section{Figura 6.}

Capitalización del tiempo dentro del producto. Fuente: Elaboración propia.

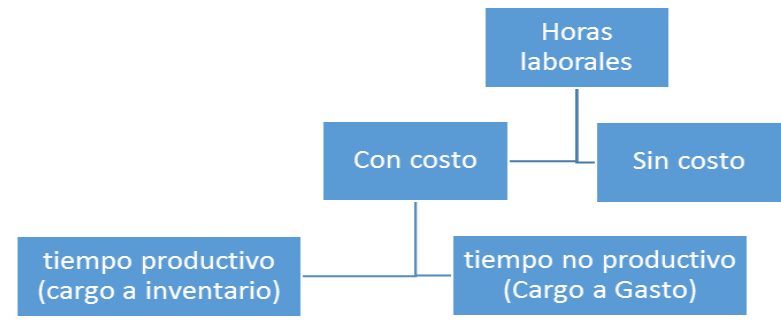

Como está expresado anteriormente, se incluirá en los costos de MOD solo lo que corresponda al importe normal, de esta manera (ver Tabla 7):

Tabla 7.

Costos de Mano de Obra.

\begin{tabular}{|c|c|c|c|}
\hline CÓDIGO & CUENTA & DEBITO & CRÉDITO \\
\hline $7205 X X$ & MOD & $X X X$ & \\
\hline $2505 X X$ & $\begin{array}{c}\text { Beneficios a empleados por } \\
\text { pagar }\end{array}$ & & $X X X$ \\
\hline
\end{tabular}

Los costos inherentes al contrato se asignarán a este rubro siempre y cuando pertenezcan al personal encargado de la elaboración de los productos o la prestación de servicios; además de otros costos asociados como: auxilio de trasporte, riesgos profesionales, pensiones, cesantías, intereses, primas, cesantías entre otros (ver Ecuación 7). 


\section{Ecuación 7.}

Costos Mano de Obra.

$$
\begin{gathered}
M O D=\text { Sueldo }+ \text { auxilios }+ \text { seguridadsocial } \\
+ \text { prestacionessociales }
\end{gathered}
$$

En algunos casos las vacaciones no hacen parte del costo del inventario, ya que este tiempo se considera no productivo, pero la realidad es que las personas, independientemente del tiempo laboral devengan vacaciones, siendo este el importe utilizado por el operario para cubrir su tiempo de ausencia.

En tercer lugar, frente a los costos indirectos se deben tener en cuenta todos los cargos accesorios (ver Tabla 8) para el correcto funcionamiento del método; estos son aquellos que se dan por riesgo, por longitud, por participación, por consumo, entre otros.

Tabla 8.

Costos indirectos asociados a cargos accesorios.

\begin{tabular}{llll}
\hline CÓDIGO & \multicolumn{1}{c}{ CUENTA } & DEBITO & CRÉDITO \\
& & & \\
\hline $73 X X X 1$ & CIF & & \\
& & XXX \\
\hline $2335 X X$ & $\begin{array}{l}\text { Costos y gastos por } \\
\text { pagar }\end{array}$ &
\end{tabular}

Fuente: Elaboración propia.

Es conveniente aclarar que es de gran dificultad separar los costos fijos de los variables, pues en este proceso debe primar la optimización del tiempo, lo que impide realizar la evaluación por celda y su clasificación uno a uno. Esto mismo ocurre con las cuentas, pues se generan condiciones para que ocurran errores de digitación.

Por otra parte, las facturas contienen un solo rubro y los costos vienen determinados en 2 conjuntos: semifijos y semivariables; esto indica que ese rubro debe ser separado. La opción más apropiada es el método de los mínimos cuadrados o también denominada regresión lineal (ver Ecuación 8) (Gutiérrez, 2014).

Ecuación 8. Regresión lineal, donde (a) son los cargos fijos y (b) los cargos variables.

$$
y=a+b x
$$

\subsubsection{Proceso de cierre}

Un proceso de cierre debe llevarse a cabo, teniendo presente que las variaciones no interfieren pero que deben separarse. A partir de todos los elementos expuestos anteriormente, se pueden separar cada uno de ellos, con el propósito de hallar la cantidad transferida y la porción que queda en proceso (ver Tabla 9).

\begin{tabular}{|c|c|c|c|c|c|}
\hline ELEMENTO & $\begin{array}{l}\text { COSTO } \\
\text { REAL }\end{array}$ & CANTIDAD & $\begin{array}{c}\text { VALOR } \\
\text { UNITARIO }\end{array}$ & $\begin{array}{c}\text { CANTIDAD } \\
\text { TRANSFERIDA }\end{array}$ & $\begin{array}{c}\text { PORCIÓN } \\
\text { EN } \\
\text { PROCESO }\end{array}$ \\
\hline MP & $x$ & $a$ & $\mathrm{x} / \mathrm{a}$ & & \\
\hline MOD & $y$ & $b$ & $y / b$ & & \\
\hline $\mathrm{CIF}$ & $Z$ & C & $z / c$ & & \\
\hline Total & & & & & \\
\hline
\end{tabular}

Tabla 9.

Entregable del proceso de cierre de contabilidad.

Fuente: Elaboración propia.

Donde $a$ estaría basado en la producción equivalente como afirma ("Costos y presupuestos," 2014), el cual propone agrupar proporcionalmente los costos de producción con los elementos que quedaron sin transferir de cada proceso (ver Ecuación 9).

\section{Ecuación 9.}

Producción equivalente

$$
a=\text { undfabricadas }+ \text { Undenprocesox } \% \text { deavance }
$$

La producción equivalente está dada a criterio del encargado de la producción, pues es quien tiene mayor conocimiento del proceso y del producto y tiene la capacidad de aumentar las unidades a producir. En este punto, ya es clara la manera como debe calcularse el valor unitario de cada elemento (ver Ecuación 10):

Ecuación 10. Valor unitario.

$$
\text { VrUnitario }=\frac{\text { Costoreal }}{\text { Qequivalente }}
$$




\section{EL MÉTODO DE COSTOS ESTÁNDAR ALINEADO A PEQUEÑAS INDUSTRIAS MANUFACTURERAS DEL ÁREA METROPOLITANA DEL VALLE DE ABURRÁ}

José Luis Cardona Olaya - Cristian Pabón Salazar - Sandra Milena Velásquez Restrepo - Carolina Abril Carrascal - Lesly Lisbeth Gómez Echeverry

Además del valor de la producción transferida (ver Ecuación 11):

Ecuación 11. Valor de la producción transferida.

$$
\text { V.Transferida }=\text { VrUnitarioxUndaPt }
$$

Donde $V r$ Unitario, corresponde al valor obtenido en la ecuación 9 y Und a Pt representa las unidades enviadas a producto terminado, se calculará la valoración de los productos en proceso con ayuda de la Ecuación 12:

Ecuación 12. Valoración de los productos en proceso.

$$
\text { CostoReal - V.Transferidas }
$$

Por otra parte, se debe ajustar la contabilidad al costo real ya que no es prudente manejar un estimado, para esto se les dará valor a las variaciones o las cantidades, al inventario de producto terminado y al costo de la mercancía vendida (ver Tabla 10).

\section{Tabla 10.}

\begin{tabular}{|c|c|c|c|}
\hline CÓDIGO & CUENTA & DEBITO & CRÉDITO \\
\hline 7199 & $\mathrm{MP}(\mathrm{A})$ & $X X X$ & \\
\hline 7105 & MP real & & $\overline{X X X}$ \\
\hline 7299 & $\mathrm{MOD}(\mathrm{A})$ & $X X X$ & \\
\hline 7205 & MOD real & & $X X X$ \\
\hline $7399 \times 1$ & CIF Fijo(A) & $X X X$ & \\
\hline $7399 \times 2$ & CIF Variable(A) & $\mathrm{XXX}$ & \\
\hline $73 X X$ & CIF reales & & $X X X$ \\
\hline $61 X X$ & CMV & $X X X$ & \\
\hline 1430 & $\begin{array}{l}\text { Inventario de Producto } \\
\text { terminado }\end{array}$ & $\overline{X X X}$ & \\
\hline 1410 & $\begin{array}{l}\text { Inventario de producto en } \\
\text { proceso }\end{array}$ & $\overline{X X X}$ & \\
\hline
\end{tabular}

Cuentas para ajustar la contabilidad al costo real.

Fuente: Elaboración propia.

Basado en el cuadro anterior, el proceso de cierre comprende los saldos de lo contabilizado durante el periodo, sin olvidar la distribución de la variación entre lo real y lo aplicado en cada uno de los rubros contables (ver Ecuaciones 13 y 14).

\section{Ecuación 13.}

Costo de mercadería vendida.

CMV

$=\frac{(\text { CostosReales }- \text { Costosaplicados }- \text { CostoPenP }) x \text { Undvendidas }}{\text { UndaPterminado }}$

\section{Ecuación 14.}

Inventario de producto terminado.

InvdePtr

$=\frac{(\text { C.Reales }- \text { C.aplicados }-C . P e n P) x(\text { UndaPT }- \text { UndVendidas })}{U n d a P T}$

Si la fórmula da como resultado un número positivo, significa que aumenta el Costo de mercadería vendida CMV y el inventario de producto terminado; entonces la variación se calificará como desfavorable; en caso contrario la variación es favorable en su totalidad. Desde el punto de vista individual, las variaciones se deberán analizar con base en situaciones económicas externas y de eficiencia interna. Cabe anotar que el CMV debe coincidir con el costo de las ventas del periodo representado en el estado de resultados anual o de periodos intermedios.

Terminado el proceso de cierre, se elabora un informe de costos denominado: Estado de Costos, contemplado en el Art. 24 y 27 del Decreto 2649 de 1993 así: "Son estados de costos aquellos que se preparan para conocer en detalle la erogaciones y cargos realizados para producir los bienes 0 prestar los servicios de los cuales un ente económico ha derivado sus ingresos." (Colombia, 1993). Existen dos opciones para ejecutar esta tarea, en primer lugar si la empresa ya realizó el ajuste de la contabilidad es posible determinar el estado de costos basados en el real (ver Figura 7).

\section{Figura 7.}

\begin{tabular}{|c|c|}
\hline \multicolumn{2}{|c|}{ ESTADO DE COSTOS } \\
\hline $\begin{array}{l}\text { MP } \\
\text { MOD } \\
\text { CIF }\end{array}$ & $\begin{array}{l}A \\
B \\
C \\
\end{array}$ \\
\hline $\begin{array}{l}\text { (=) C producción } \\
\text { (+) IIPP }\end{array}$ & $D$ \\
\hline $\begin{array}{l}\text { (=) C. Dpto. en P } \\
\text { (-) IFPP }\end{array}$ & $E$ \\
\hline $\begin{array}{l}\text { (=) C. Dpto. term } \\
(+) \mathrm{IIPT}\end{array}$ & $\boldsymbol{F}$ \\
\hline $\begin{array}{l}\text { (=) CPDV } \\
\text { (-) IFPT }\end{array}$ & $\boldsymbol{G}$ \\
\hline $\begin{array}{l}\text { (=) CMV } \\
\text { VARIACIONES }\end{array}$ & $\boldsymbol{H}$ \\
\hline CMV TOTAL & \\
\hline
\end{tabular}

Estado de costos sin variaciones.

Fuente: Elaboración propia. 
Para elaborar este estado se debe tener las siguientes consideraciones

a) Corresponde al saldo de MP real del cual se puede extractar de la cuenta 71

b) Corresponde al saldo de MOD real del cual se puede extractar de la cuenta 72

c) Corresponde al saldo de los CIF reales del cual se puede extractar de la cuenta 73

d) Corresponde al saldo inicial de la cuenta 1410 (Inventario de productos en proceso).

e) Corresponde al saldo final de la cuenta 1410 (Inventario de productos en proceso)

f) Corresponde al saldo inicial de la cuenta 1430 (Inventario de productos terminados).

g) Corresponde al saldo final de la cuenta 1430 (Inventario de productos terminados)

El otro modelo corresponde al estado de costos basado en los estándares, reconociendo al final la variación total el cual ajusta al CMV y a todos los demás conceptos (ver Figura 8). Se recomienda verificar la existencia de un margen de error en el presupuesto, lo cual se puede analizar estudiando la variación económica o de eficiencia.

Figura 8.

Estado de Costos basado en estándares.

\begin{tabular}{|c|c|}
\hline \multicolumn{2}{|c|}{ ESTADO DE COSTOS } \\
\hline MP & $\boldsymbol{A}$ \\
\hline MOD & $\boldsymbol{B}$ \\
\hline CIF & $C$ \\
\hline (=) C producción & \\
\hline (+) IIPP & $D$ \\
\hline (=) C. Dpto. en P & $E$ \\
\hline $\begin{array}{l}\text { (-) IFPP } \\
(=) \text { C. Dpto. term }\end{array}$ & \\
\hline (+) IIPT & $\boldsymbol{F}$ \\
\hline (=) CPDV & \\
\hline (-) IFPT & $\boldsymbol{G}$ \\
\hline (=) CMV & $\bar{L}$ \\
\hline
\end{tabular}

Fuente: Elaboración propia.

Para elaborar este estado se debe tener las siguientes consideraciones

a) Corresponde al saldo de MP Aplicada del cual se puede extractar de la cuenta 7199

b) Corresponde al saldo de MOD Aplicada real del cual se puede extractar de la cuenta 7299

c) Corresponde al saldo de los CIF Aplicados del cual se puede extractar de la cuenta 7399

d) Corresponde al saldo inicial de la cuenta 1410 (Inventario de productos en proceso) e) Corresponde al saldo final de la cuenta 1410 (Inventario de productos en proceso)

f) Corresponde al saldo inicial de la cuenta 1430 (Inventario de productos terminados)

g) Corresponde al saldo final de la cuenta 1430 (Inventario de productos terminados)

Las variaciones serán el resultado de las cuentas 71,72 y 73 reales menos las 7199,7299 y 7399 , dando así un resultado desfavorable aumentando el CMV o por el contrario un resultado favorable disminuyendo el CMV.

Esta adaptación que partió de las Normas Internacionales de Contabilidad, permite estandarizar las regulaciones, normas y procedimientos contables para que las cifras puedan compararse a nivel internacional dentro del sector del cuero, el calzado y su manufactura; esto facilitará procesos de importación y exportación, tanto de insumos y maquinaria, como de productos fabricados.

\subsection{Prueba piloto}

Según los resultados de la encuesta de satisfacción, se confirmó la importancia de la implementación del método de costos estándar en las empresas del sector del cuero, calzado y su manufactura; además, se obtuvo una percepción positiva, calificándolo como un método confiable que la empresa recomendaría implementar a otras organizaciones. Además, la encuesta evidenció que la mayoría de empresas fabriles no invierten en investigación y desarrollo, puesto que aumenta el costo de sus productos y por lo tanto, genera una ventaja competitiva menor. Cabe aclarar que las empresas protegen la información de sus productos, por lo que en este trabajo no se mostraron datos del producto real sino simulados para lograr una mejor comprensión en el lector y respetando los derechos de los productos que se elaboran en la empresa estudiada.

Al determinar el costo unitario de los productos, el área contable obtuvo los datos de costos necesarios para la planeación de las operaciones de manufactura y el control de los costos de producción para aumentar la ventaja competitiva de la empresa y es oportuna para tomar de decisiones estratégicas, relacionadas con inversiones a largo y mediano plazo.

Además, es importante que la información que reposa en estos informes debe ser comparable entre varios periodos de tiempo para que ésta sea de real utilidad para quienes la utilicen. Esto ayudará a identificar el comportamiento de la situación financiera de la entidad: su rendimiento y flujos de efectivo. 


\section{EL MÉTODO DE COSTOS ESTÁNDAR ALINEADO A PEQUEÑAS INDUSTRIAS MANUFACTURERAS DEL ÁREA METROPOLITANA DEL VALLE DE ABURRÁ}

José Luis Cardona Olaya - Cristian Pabón Salazar - Sandra Milena Velásquez Restrepo - Carolina Abril Carrascal - Lesly Lisbeth Gómez Echeverry

\section{CONCLUSIONES:}

Fue posible concluir que el método de costos estándar es una herramienta de fácil acceso a su manejo; no obstante requiere de un control adecuado de los costos que permita estar al tanto de sus variaciones.

Además, gracias a la implementación de la prueba piloto, fue posible concluir que la información suministrada mediante este método puede ser catalogada como insumo para tomar decisiones estratégicas en las empresas; ya que enseña estados contables en tiempo presente y esto facilita la implementación de planes de acción inmediatos.

El desconocimiento del método es una de las principales razones por las cuales éste no es implementado en estas empresas manufactureras; sin embargo existe gran disposición de las mismas para incluirlo en su sistema de costeo y así comprobar sus beneficios. Por último, también se logró concluir que es factible implementar este método en el sector del cuero, calzado y su manufactura, al obtener resultados satisfactorios luego de su utilización.

\section{REFERENCIAS:}

\section{REFERENCIAS BIBLIOGRÁFICAS}

Blanco, M. (2015). Costes para la toma de Decisiones.Madrid: CEF (Escuela de Negocios).

Checo Paulino, E. M. (2000). Costos estándar aplicados al establecimiento de los estándares de los tres elementos básicos del costo. El Cid Editor | apuntes.

Colombia, P. de la R. (1993). Decreto Numero 2649 de 1993, 1993 (diciembre 29).

Costos y presupuestos. (2014). Huancayo: Universidad peruana de los Andes. Disponible desde <http://181.49.158.75/blogsuts/costosypresu puestos/files/2014/05/COSTOS-YPRESUPUESTOS1.pdf>

Duque, M. I., Osorio, J. A., y Agudelo, D. M. (2012). Análisis de la aplicación del sistema de costeo estándar en las empresas manufactureras colombianas. Revista Del Instituto Internacional de Costos, 97-123.
Gómez, O. (2005). Contabilidad de costos. McGraw Hill.

Horngren, C. T., Foster, G., y Datar, S. (2007). Contabilidad de costos: un enfoque gerencial. México: Pearson education.

Madegowda, J. (2007). Management accounting.

Norma Internacional de Contabilidad 2. (NIC 2). (s.f.).

Ochoa, C. (2013). ¿Qué tamaño de muestra necesito? Disponible desde <http://www.netquest.com/blog/es/quetamano-de-muestra-necesito/>

Rao, A., Kashanni, H., y Marie, A. (2010). Analysis of managerial efficiency in insurance sector in the UAE: an emerging economy. International Journal of Managerial Finance, 6(4), 329-343. Disponible desde $<$ <ttps://doi.org/http://dx.doi.org/10.1108/174 39131011074477>

Salguero, K. (2011). Diseño de un sistema de costos estándar para la empresa Confecciones Macar Ltda. Universidad de la Salle.

Sinisterra, G. (2006). Contabilidad de costos (Ecoe Edici). Bogotá.

Strengthening cost competitiveness. (2012). Kolkata: The institute of cost accountants of India.

Universidad Eafit, C. contable. (s.f.). Tipos de costos indirectos de acuerdo a su comportamiento. Disponible desde $<$ <ttp://www.eafit.edu.co/escuelas/administra cion/consultoriocontable/Documents/nota.pdf> 